\title{
Preparation and Characterization Challenges in Hydroxylation of Neoprene Rubber
}

\author{
Amr S Ismail* and Eid A Ismail \\ Petrochemicals department, Egyptian Petroleum Research Institute, Egypt \\ *Corresponding author: Ismail AS, Petrochemicals department, Egyptian Petroleum Research Institute, 11727 Nasr City, Cairo, Egypt.
}

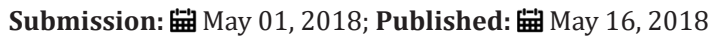

\begin{abstract}
Attempts for hydroxylation of the synthetic polychloroprene rubber (neoprene) were employed. In this paper, high concentrated aqueous solution of sodium hydroxide was used to introduce $\mathrm{OH}$ groups into liquid polychloroprene rubber via direct hydroxylation process at ambient conditions. Structure and composition of neoprene rubber before and after the hydroxylation process were investigated by Fourier transform infrared spectroscopy (FTIR) and X-ray diffraction (XRD)techniques. It is show that there are changes in crystallinity and agglomeration of rubber due to influence of the strong alkaline medium. Moreover, the morphological observations show that the modified neoprene layers more harder than the unmodified one. Based on the analytical results and the experimental data, it can develpneoprene rubber for various applications.
\end{abstract}

Keywords: Neoprene; Polychloroprene; Hydroxylation; Sodium hydroxide

\section{Introduction}

Neoprene rubber is a synthetic rubber composed of polymerized chloroprene, and is generally referred to as polychloroprene. Polychloroprene is primarily composed of carbon, hydrogen and chlorine polymers which are cross-linked to give neoprene specific desirable characterizations such as chemical inertness, thermal, water, oil and solvent resistance. Moreover, neoprene rubber resists deterioration or degradation more than natural rubber. This relative properties makes it well suitable for useful applications such as gaskets, hoses and corrosion-resistant coatings [1]. It can be used as a base for adhesives, noise isolation inpowertransforming techniques and as padding in external metal cases to protect the contents while allowing a snug fit. Owing to the chlorine based neoprene structure as shown in Figure 1, it has a fire

retardant effect better than several hydrocarbons based rubbers resulting in its appearance in weather stripping for fire doors and in combat related attire such as gloves and face masks. Neoprene has a tolerance of extreme conditions, it is used to line landfills. Without changing its main structure, neoprenecan be found in many applications $[2,3]$. There are few research articles reported on the modification of natural and synthetic rubber compared with other works related long chain hydrocarbons. Although natural polyisoprene rubber has a good mechanical characterizations for many useful implementations, it exhibits low resistance towardsthe long environmental attacks [4-6]. Wherefore, there are widely possibilities to develop and improve the rubberperformance to obtain new functional propertiessuch as epoxidation and hydroxylation based on the functional group content $[7,8]$.

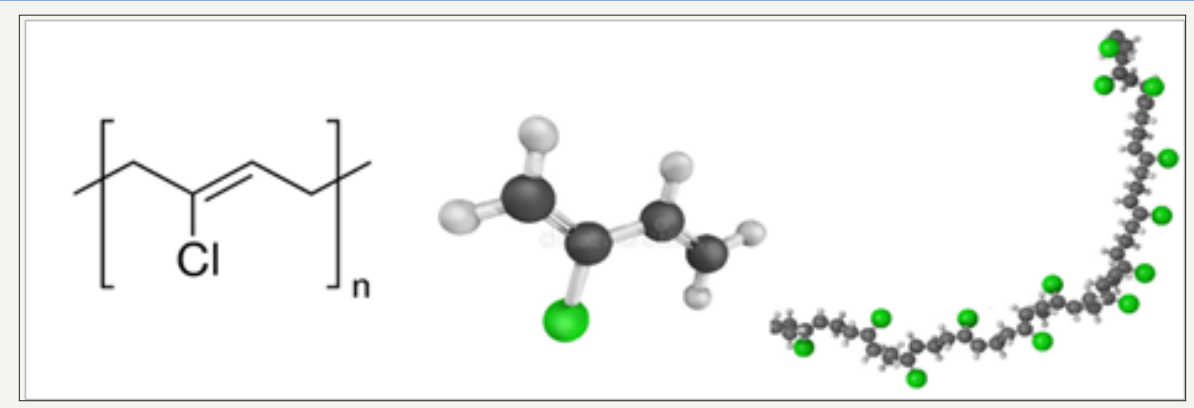

Figure 1: Chemical structure of synthetic neoprene rubber. 
Hydroxylation of neoprene is very reactive owing to the hydroxyl groups influence in the long polymer chain. Therefore, hydroxylation of liquid polychloroprene rubber as shown in Figure 2 can acts as intermediate processes for several applications such as modifier, coating, adhesive,compatibilizer, tackifier and plasticizer. This research reports hydroxylation of neoprene rubber using high concentrated aqueous sodium hydroxide solutionto give modifiedneoprene. Neoprene and modifiedneoprenes were analyzed using Fourier transform infrared spectroscopy (FTIR) and X-ray diffraction (XRD) techniques. The influence of hydroxyl group on rubber structure was investigated.<smiles>CCC=C(Cl)CCC=C(Cl)CCC=C(Cl)CC</smiles>

Neoprene

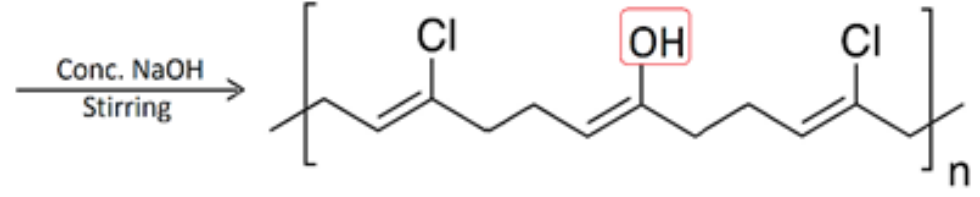

Hydroxlated Neoprene

Figure 2: Hydroxylation process of synthetic neoprene rubber.

\section{Materials and Methods}

\section{Materials}

Neoprene rubber was obtained from the Bahrain Rubber Company (BRC) W.L.L, Kingdom of Bahrain. The neoprene samples were stored under ambient conditions until use.

\section{Methods}

Hydroxylation of neoprene rubber: Prior to use, $1 \mathrm{~g}$ of solid neoprene rubber was dissolved completely in $70 \mathrm{ml}$ carbon tetrachloride solvent in glass vessel for 2 days with continuous stirring. 50\% aqueous sodium hydroxide solution was prepared based on the fundamental procedures. Drop wise addition of alkaline solution to the liquid neoprene rubber under ambient condition with continuous stirring. The hydroxylation process revealing a yellowish-whiteviscous solution. The obtainedsolution which mainly based on hydroxylatedrubber was poured in a petri dish for curing. The OHeffectin the building block of synthetic neoprene rubber was investigated.

\section{Results and Discussion}

\section{Characterization}

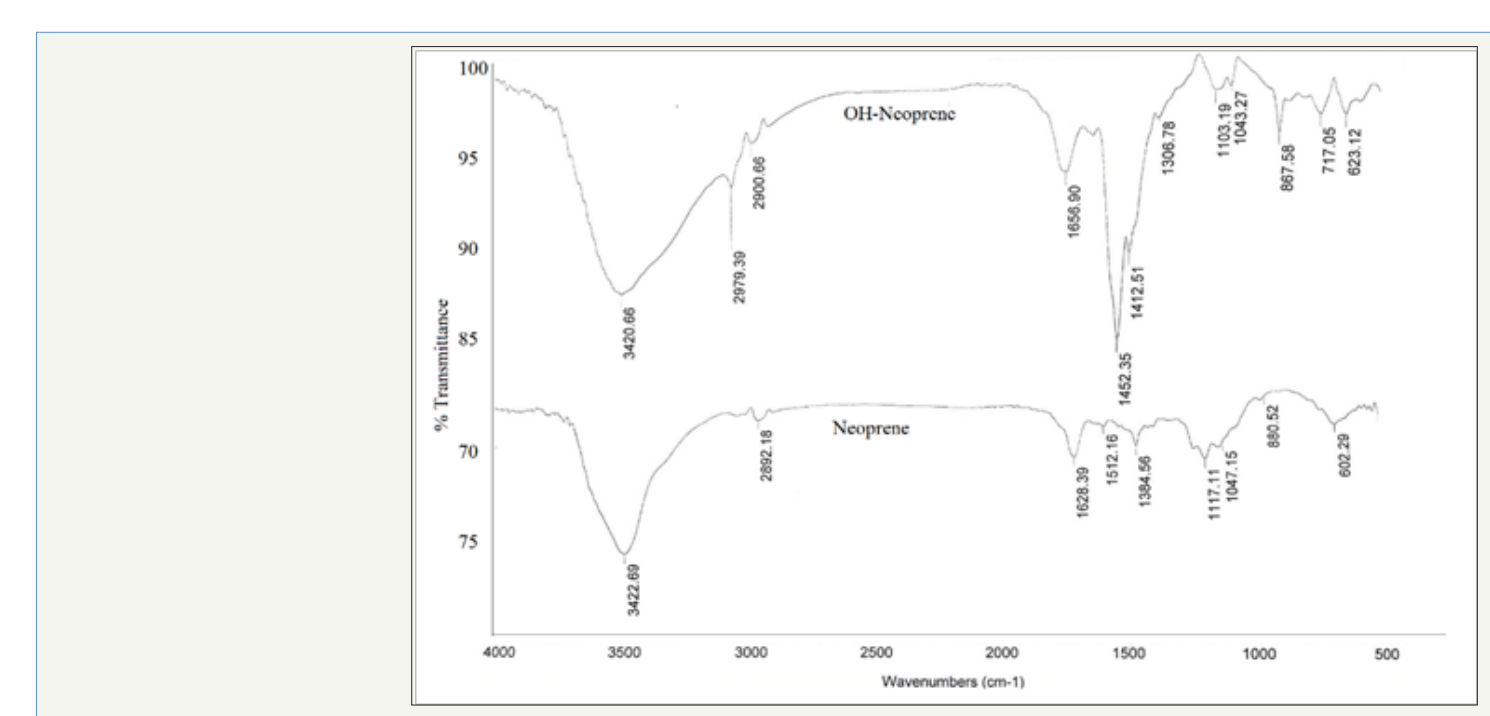

Figure 3: FTIR patterns for modified and unmodified neoprene rubber.

Fourier transform infrared spectroscopy (FT-IR): Fourier transform infrared spectroscopy (FT-IR) is a technique which is used to obtain an infrared spectrum of absorption, emission, photoconductivity or Raman scattering of a solid, liquid or gas. An FT-IR spectrometer simultaneously collects spectral data in a wide spectral range. FT-IR spectra analysis was obtained using ATI Mattson model Genesis Series (USA) infrared spectrophotometer adopting $\mathrm{KBr}$ technique. For all samples, the $\mathrm{KBr}$ technique was carried out approximately in a quantitative manner since the weight of sample and that of $\mathrm{KBr}$ were always kept constant (JASCO, V570, UV/VIS Spectrophotometer, made in Japan).

X-ray diffraction (XRD): X-ray diffraction (XRD) patterns were recorded in the range $2 \theta=4-80^{\circ}$, using Philips Powder Diffractometer with $\mathrm{Cu} \mathrm{K} \alpha 1$ radiation. The instrument was operated at $40 \mathrm{KV}$ and $40 \mathrm{~mA}$. The spectra were recorded at scanning speed of $2^{\circ}$ in $2 \theta / \mathrm{min}$. The $2 \theta$ and the relative intensity I/Io were deduced from the chart and the corresponding d-values calculated from the Bragg's equation. 
FTIR diagrams of the modified and unmodified neoprene rubber are given in Figure 3. From the diagrams, it can be clearly seen that there are absorption bands of streching $\mathrm{C}=\mathrm{C}$ groups at $1680 \mathrm{~cm}^{-1}$, where attributed to the alkene structure. Weak broad absorption bands of streching C-H groups appeared at $2892 \mathrm{~cm}^{-1}$. For modified rubber, two absorption bands observed at $2979 \mathrm{~cm}^{-1}$ and $2900 \mathrm{~cm}^{-1}$ due to the rich $\mathrm{CH}$ - and $\mathrm{CH}=$ groups on the chemical structure of neoprene. There are significant broading variations in absorption bands oftreated rubber samples at $3422 \mathrm{~cm}^{-1}$ and $1412 \mathrm{~cm}^{-1}$ related to $\mathrm{OH}-\mathrm{C}$ streching and binding. This behaviour may attribute to the OHgroup based neoprene rubber rather than unmodified one [9].
X-ray diffractograms of modified and unmodified rubber reveals the appearance of sharp and high intensities $31.49 \%$, $100 \%, 32 \%)$ and $(45.42 \%, 51.23 \%, 47.41 \%)$ diffractions lines for modified rubber sample at d-distances $2.84,3.4$ and $3.2 \AA$ and 1.99 , 1.8 and $1.7 \AA$ as shown in Figure 4. These lines are the main lines characterizing $\mathrm{OH}$, according to ASTM 89-0598 and ASTM 24-1148, respectively. Crystallite size is mainly calculated using Scherrer equation to the peaks at $2 \theta=31.49$ and $2 \theta=45.42$. The unmodified neoprene diffractogram reveals weak inrtensity $(28.54 \%, 100 \%$, $31.76 \%$ ) diffraction line at d-distances 3.12, 3.89 and $3.69 \AA$ [10].

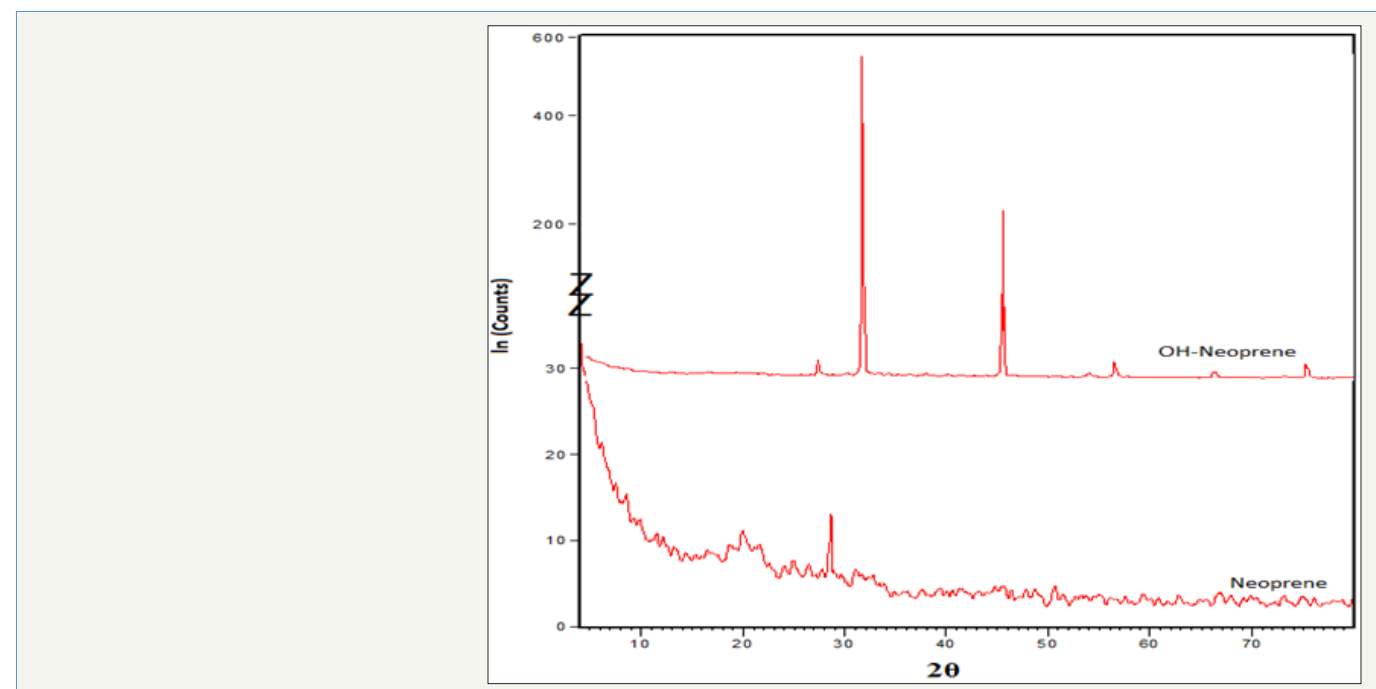

Figure 4: X-ray diffraction of modified and unmodified neoprene rubber.

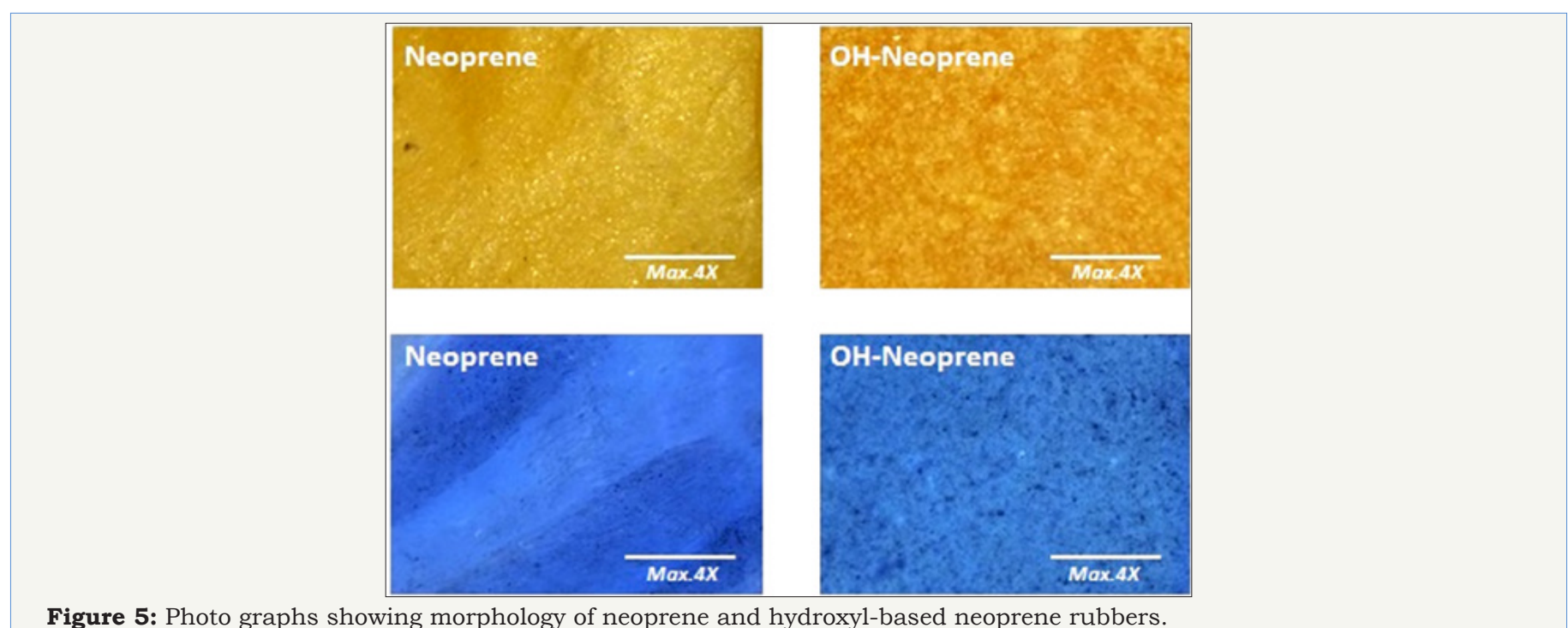

Figure 5: Photo graphs showing morphology of neoprene and hydroxyl-based neoprene rubbers.

Neoprene rubber morphology was exhibit coalescence and featureless shape due to agglomiration performance at room temperature. It revealed tacky structural countenance, but at presence of hydroxyl group content, dense graphs were obtained as shown in Figure 5. We have found that pure neoprene layers are featureless and has sticky behaviour, while after hydroxylation process it show significant retention stability of the layer shape. These new characterizations based on hydroxyl group contents manifest an increase in surface hardness and thermal resistance of the modified rubber [11].

\section{Conclusion}

Attemptes were employed for hydroxylation of liquid neoprene rubber using high concentration aqueous sodium hydroxide solution. FTIR and XRD spectra show the composition and the crystallinity of modified and nonmodified neoprene rubber which 
reduced greatly due to the chelation. The characterizations show that there are changes in agglomeration of rubber due to influence of the strong alkaline $\mathrm{OH}$ groups. Furthermore, the casted hydroxyl based neoprene layers appeared harder than the pure one. Based on these investigations and observations, it can develp and improve synthetic neoprene rubber for differentimplementations.

\section{Acknowledgement}

Financially support from the Egyptian Petroleum Research Institute (EPRI) is greatly appreciated.

\section{References}

1. Bridgewater E (1940) Neoprene, the chloroprene rubber. Industrial and Engineering Chemistry 32(9): 1155-1156.

2. Smith JK (1985) The ten-year invention: Neoprene and du pont research, 1930-1939. Technology and Culture 26(1): 34-55.

3. Paciorek KL, Kratzer RH, Kaufman J, Nakahara J, Harstein AM (1975) Thermal oxidative decomposition studies of neoprene compositions. Am Ind Hyg Assoc J 36(1): 10-16.

4. Low KO, Teo WC (2012) Characteristics of SBR, Neoprene and EPDM compounds in a single-pass pendulum scratch.Tribology International 54: 9-16.
5. Baah CA, Baah JI (2001) Effect of organic reagents on neoprene, nitrile and natural rubbers. Materials and Design 22(5): 403-405.

6. Yelvington M, Brown S, Castro MM, Nick TG (2013) The use of neoprene as a scar management modality. Burns 39(5): 866-875.

7. Azhar NHA, Rasid HM, YusoffS FM (2016) Chemical modifications of liquid natural rubber. AIP Conference Proceedings 1784: 030024.

8. Surya I, Maulina S, Ismail H (2018) Effects of alkanolamide and epoxidation in natural rubber and epoxidized natural rubbers compound. IOP Conference Series: Materials Science and Engineering 299: 012061.

9. Ismail AS (2015) Pervaporative dehydration of alcohol-water mixtures using hydrophilic polyelectrolyte chitosan-sodium carboxymethyl cellulose membranes. International Journal of Science, Engineering and Technology 3(5): 1344-1351.

10. Maciel JS, Silva DA, Paula HCB, Paula RCM (2005) Chitosan carboxymethyl cashew gum polyelectrolyte complex: synthesis and thermal stability. European Polymer Journal 41(11): 2726-2733.

11. Burford P, VO CD (1999) Morphology development in polychloroprenepolystyrene latex interpenetrating polymer networks. Journal of Applied Polymer Science 74: 629-638.
Creative Commons Attribution 4.0

International License

For possible submissions Click Here

\section{Submit Article}

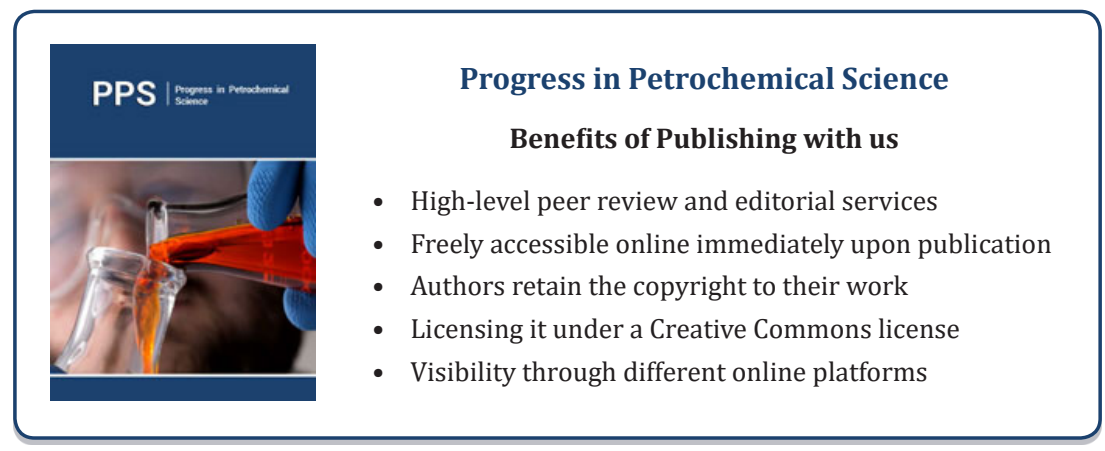

